\title{
Eğitimde Drama ve Sosyal Değişim: Teorik Bakış Açıları*
}

\author{
$M^{\text {a }}$. Rosario NAVARRO \\ Sevilla Üniversitesi
}

\begin{abstract}
Özet
Ĕ̈itimde drama, gerçek durumları tanımlamayı sağlayan bir araçtır, bu durumların saptanmast diğer eğitim yöntemleriyle zordur. Özellikle de, drama sürecine giren herkesin kişisel ve sosyal gelişimi için çok güçlü bir araç hâline gelir. Bu makalede çocuklarda, gençlerde, yetişkinlerde kişisel ve müşterek sosyal sorumluluğu teşvik etmede yaratıcı dramanın nasıl etkili olabileceğini tartıştık. Örneğin, tamamen demokratik olan adil bir dünya için vatandaşlık ĕ̆itimi kavramı bunlardan birisidir. Ancak, yaratıcı dramanın temel eğitim teknikleriyle dar bir alanda kullanılmass yaygındır. Bu makalede dramayla sosyal gelişim, sosyal değişimin teorik açılardan ilişkilerinin kilit noktalarını tartışıyoruz. Ayrıca dramayla bağlantılı-son derece iyi tanımlanmış ve böylece dikkatli bir eğitim gerektiren-teknikler bütün eğitim pratiğini tümüyle destekleyen amaçların esaslı konuları ve değerleriyle yakından ilişkilidir.
\end{abstract}

Anahtar Sözcükler:Eğitimde drama, sosyal sorumluluk, yaratıcılık, grup değişimi ve sosyal değişim.

\begin{abstract}
Drama in education is a tool that allows the explanation of aspects of reality that are difficult to address with other educational methods. In particular, it becomes a powerful tool for personal and social development for all those involved. Here we discuss the possibilities of drama for promoting personal and collective social responsibility in children, young people and adults, for example, training in citizenship for a just world that is truly democratic. However, it is common for drama to be used with scant attention to any underlying educational techniques. Here we discuss some key issues that show some theoretical aspects of drama in relation to social development and social change and how its associated techniques-which are very well-defined and thus require careful training-are closely related to fundamental subjects of the aims and values that underpin the whole practice of education.
\end{abstract}

Keywords: Drama in education, social responsibility, creativity, group and social change.

\section{Eğitim Hakkında Düşünmek: Amaçların Önemi}

Eğitimciler olarak biz, davranışımızı belirleyen değerleri, bir insan olarak görüşümüzü, aradığımız gerçeklik vizyonunu, yanıt vermek için aradığımız ve gerçekleştirmeye çalıştığımız hedeflerimizi ve tüm bunlara karşı olan duruşumuzu tanımlamalıyız. Herkes gerçekle ilgili bir bakış açısı seçer ve bu da bizim davranışlarımızı belirler. Neticede biz seçtiğimiz bu gerçekliğin

\footnotetext{
* Zeynep Olgun tarafından çevrilmiştir. Atılım Üniversitesi, Okutman, E-posta: ikikiloelma@gmail.com

${ }^{1}$ Doç.Dr.,Sevilla Üniversitesi, Eğitim Bilimleri ve Felsefe alanlarında derece sahibi. Doktora tezi, Eğitimde Dramanın Pedagojik Değeri: Öğretmen Eğitiminin Başlangıcındaki Önemi. (Bu tez, Sevilla Üniversitesi tarafından verilen Avrupa Doktorası unvanını ve 2005 yılının en iyi doktorası ödülünü almıștır) rosanavarro@us.es
} 
öğrencilerimiz ve toplum için doğuracağı sonuçların farkında olmak zorundayız.

Bu yüzden her eğitim eylemi bir felsefi alt yapıya ihtiyaç duyar ve desteklendirilir, bu felsefi alt yapı da bizim öğretme görevimizi yönlendiren bir dizi kuralla yakından ilişkilidir. Bu felsefi alt yapılar bizim belirli yöntemleri seçmemize sebep olan ve kendimiz için belirlediğimiz amaçlarımıza ulaşmamız için gerekli olan çerçeveyi oluşturur. Diğer taraftan, koyduğumuz amaçların eğitim aktivitemizi düzenlemekte doğrudan etkisi vardır. Bu sebepledir ki, tarafsız eğitim diye bir şey asla yoktur, bunun yerine her zaman bir amaç ve hedef vardır (Núñez and Romero, 2003).

Diğer eğitsel yöntemlerde olduğu gibi eğitimde dramanın kullanımı da tarafsız değildir. Onu bir yöntem olarak kullanan kişinin (liderin) niyetine, insanlığı nasıl algıladığına olduğu kadar çalışmanın yapıldığg kültüre de bağlıdır. (Neelands, 2004; Motos, 2006).

$\mathrm{Bu}$ yüzdendir ki karşılık verdiğimiz ve argümanlarımızla amaçlarımızı desteklediğimiz "gerçekliğin" nesnelleştirilmeye çalışılması son derece önemlidir.

Bu makalede İngiliz akımlarını eğitimde yaratıcı drama ile kullanarak, bizim çok temel, önemli olduğunu düşündüğümüz bir kişinin tüm eğitiminde yaratıcı dramanın kullanımı üzerine dikkat çekmeye çalıştık, ki bir kişinin tüm eğitimi demek esasında eğitsel görevin temel amacıdır.

\section{Ĕ̈itsel Amaçlar ve Ĕ̆itimde Kişisel Ĕ̆ilimler}

Biz, bireyleri izole varlıklar olarak değil de özgür, sosyal, aşkın varlıklar olarak görüyoruz ve böylece onlar sadece birer nesne olmaktan çıkıyorlar. Hiç şüphesiz her bireyin sahip olduğu “insan onurunun bozulmaz değeri” fikri, Batı kültüründe somut gerçeklerle sorgulanan bir durum. Dahası, anlıyoruz ki özgürlük ve sorumluluk birbiriyle el ele giden kavramlardır. Mounier'a göre özgürlük (Bernabeu, 2006), bir taraftan bireysel sorumluluk gerektirir, ancak diğer taraftan özgürlük, diğer insanlara kaçınılmaz bir bağlılık anlamına gelir. Bu yüzden ne kadar özgür olursak insan doğamızla uyum içinde olan seçenekleri o kadar kolay seçebiliriz ki bu seçeneklerden biri dediğer insanlara ihtiyaç duymamızı ve onlara kendimizi adamamızı sağlayan sosyal varlı̆̆ımızdır.

Kısacası özgürlük; onur, sosyal doğamız, diğer insanlara karşı olan sorumluluğumuz ve adanmışlığımız, kimliğimizin üzerine inşa olduğu temel dayanaklardandır, bunları önemsememek ya da bunlardan kaçınmak kendi doğamıza aykırı olacaktır. Kimliğimizi belirlemeye eğitimde kişisel akımlardan başlarsak (Mounier 1905-1950, Milani 1923-1967, Freire 19211997), anlarız ki bir bireyin kendini gerçekten farketmesi aynı zamanda özgürleşmesi demektir. Böylece birey, bilgi ve eylem aracılığıyla, kendisi ve dünya arasında özerk bir bilinç geliştirir 
(Bernabeu, 2006). Böylece biz toplumun doğası tarafından ortaya koyulan, eğitim önerimizde var olan eğitimdeki kişisel bakış özelliklerini sürdürürüz ki bu öneri, topluma açıktır, aktif ve eleştireldir, demokratik katılımı teşvik eder ve son olarak da tıpkı eğitimin en başından hedeflediği gibi insanların tam ve kolektif gelişimini sağlar.

Bu araştırma makalesinde; söz etmiş olduğumuz amaçlarla ilişkilenen eğitimde yaratıcı drama pratiğinin bazı temel yönlerini kısaca açıklamaya çalıştık.

\section{Eğitimi Bağlam İçinde Ele Almak}

Gerçekliğe dair yaptığımız analizler bir ölçüde eğitimde yaptığımız çalışmaları belirler. $\mathrm{Bu}$ yüzden, bu noktanın en çok üstüne düşülmesi gereken husus olduğunu düşünüyoruz. Milani, çalışmalarına öğrencilerinin dünyaya karşı ufuklarını genişletmek için onları en çok endişelendiren somut gerçeklikten başlardı. Eğitim, hayatın içinden doğmuştur; ancak kişiye ya da gruba has kalmamıştır, hatta bir bölgeye ya da ulusal bir çevreye bile ait olmamıştır ancak eğitim, sınıfı dış düyanın durumuyla birleştirir, bu da bir tutum takınmayı gerektirir. Milani’nin günlük gazeteleri sınıfta birlikte okumaya ya da tarih derslerine verdiği önem herkesçe bilinir. $\mathrm{Bu}$ belirgin aktiviteler çatışmaların, problem durumların nedenlerinin hem tarihsel hem de politik araştırmalarının ilk adımlarıdır (Barbiana Okulu Öğrencileri, 1970). Eğitimin net bir sosyal ve politik boyutu vardır. Tıpkı Freire (1990) gibi Milani de gerçekle yüzleşmeye ve onu değiştirmeye gücü olan geleceğin vatandaşlarını eğitimle yetiştirebileceğimizi dile getirir. Bu yüzden eğitime gereken önem verilmelidir. Freire ve Milani gibi, biz de her eğitsel sürecin belirli bir tarihsel anla bağlantılanması ve o dönemin içine eklenmesi gerekliliğine inanıyoruz. Biz, eğitimle insanları kontrolü ele almaları için, içinde yaşadıkları toplumla ilişki kurabilmeleri böylece de daha adil ve merhametli bir dünya kurmaları için eğitmeye çalışıyoruz.

Şu anki tarihî anımız dünyayı genel anlamda tanımlamada kullanılan anahtar kavramlardan bir tanesi olan küreselleşme sürecine koşullandırılmış durumdadır. Küreselleşmenin en çok bilinen özelliklerinden bir tanesi, dünyanın büyük bir kısmının birbirine bağlı olduğudur, en belirleyici gerçeği ise açlıktan ölen, her birinin fakirlik derecesi birbirinden düşük ve insanlığın çoğunluğunu oluşturan uçsuz bucaksız sayıdaki insan gruplarıdır. ${ }^{2}$ González Monteagudo (2003)'nun “Küreselleşmekte olan şey, gelişme değil eşitsizliktir.” deyip küreselleşmenin özelliklerini analiz ettiği fikrine katılıyoruz. Günümüz dünyasında açlık nedenli ölümler, en yaygın

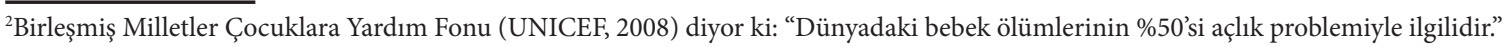


ölüm sebebidir. Bu korkunç, acımasız, insanlık dışı gerçeklik her gün gözlerimizin önünde gerçekleşiyor ve bizim vicdanımız cevaplar ${ }^{3}$ bulmakta bizi çaresiz bırakıyor. Bu aslında bu gezegenin nüfusunun çoğunluğunun içinde yer aldığı günlük bir gerçektir. Biz, dünya çapında gerçek bir sosyal sorun statüsü kazanış bir gerçekle karşı karşıyayız.

Bu dünya durumunun aksine, bizim yaşantılarımız sanki kimse acı çekmiyormuş gibi, sanki tüm bunlar hiç de bizim sorunumuz değilmiş gibi sürmekte. Bizce, her kim bu gerçekliği reddederse onun vicdan muhakemesi sahtekârdır. Her kadın ve erkek küreselleşen dünyaya, ezilen çoğunluğa karşı alacağı tavır konusunda karar verme hakkına sahiptir. Bu yüzden insanlığa karşı olan değerlerin, saygılı tutumların oluşturulabileceği, insanların kendi haklarını savunmaları için arzulu oldukları bir ortam yaratmanın önemine, bilinmeyene karşı yeni yanıtlar bulmamızı sağlayan yaratıcılığ 1 geliştirmenin de hayati önem taşıdığına inanıyoruz. Bu açıdan eğitimde drama benzer ortamların beslenmesi, ahlak ve değerlerin öğretimi için uygun bir eğitsel araçtır (Winston, 1999).

\section{Başka Bir Gerçekliğe Duyulan İhtiyaç}

Bizim amacımız sadece gerçeği anlamak değildir, aynı zamanda bu gerçekliği değiştirmenin yollarını arıyoruz. Açıklamış oluğumuz gibi sorumluluğumuzdan kaynaklanan özgürlüğümüz bizi bu kez de durumun sorumluluğunu almaya iter. Bu durumu Freire'nin pedagojisinde kullanılan bir kavramla, “Umut dolu gerçekçilik”le tanımlayabiliriz (Freire, 2005). Kötümser olmaktan bir hayli uzak olan bu durum, daha adil bir gerçekliğin inşasında aktif bir tutuma yol açar. Demek ki gerçeğin analizi, bizi onu acilen değiştirmeye ve bu değişikliğe duyulan umuda ikna eder.

Guiso Medellín (2003)'in de açıkladığı gibi, Freire umudu gerekli ama yetersiz bir unsur olarak ifade eder. Umut, tek başına dünyayı değiştiremez. Ama dünyayı değiştirmek istiyorsak da bunu umut olmadan başaramayız, değişim sürecini başlatmamız bile mümkün değildir. Ama süreç ve eylem olmadan umut bozulur ve bu durum, "trajik bir umutsuzluk" hâline gelir, umutsuzluk ise değişime direnç gösterir. Umudun, tarihsel bir gerçeklik olabilmesi için hakikatlere gereksinimi vardır (Freire, 1992, Guiso Medellín, 2003). Öğretmenlik yaptığımız kuşaklara gerçeği değiştirmekle ilgili çok derin bir umutsuzluk öğrettiğimizi sıklıkla gözlemliyoruz, çocuklar pasif olmayı, sadece izlemeyi öğreniyorlar ve bu onları doğal olarak hayatları boyunca çok büyük ölçüde etkiliyor.

Eğitim sayesinde bu gerçekliğin dönüşümünü sağlamanın yollarını arıyoruz. Belli ki, eğitimciler

${ }^{3}$ Dünya Bankası’nın eski Başkan Yardımcısı bugün Birleşmiş Milletler’in gıda danışmanı Jean Ziegler: “Biz günbegün gerçekleşen âdeta buz tutmuş umursamazlığımızın sebep olduğu kasti bir katliama karşıyız. Açlık uzun zaman önce kader olmaktan çıkmıştır. Buna göz yummak sessizce işlenen bir toplu katliamdır." beyanında bulunmuştır.(Ziegler, 2007). 
adına bu değişimi hem sınıfta hem de dış dünyada destekleyecek net bir seçim gereklidir.

\section{Drama ve Dönüşüm}

Sosyal varlıklar olarak doğamız bizi yakın ya da uzak gerçeklik hakkında sorumluluk almaya iten temel bir karakteristiğe sahiptir. Eğitimde dramanın dönüştürücü gücü bir taraftan birey üzerinde diğer taraftan çevre üzerinde yol açtı̆̆ı değişikliklerden kaynaklanır; kişinin ifadesini özgürleştirir, kişinin kendine güvenini artırı, diğerlerine güven kazanmasını sağlar, dinleme ve tartışma becerilerini geliştirir, diğer yandan ise bireyin değişmesiyle çevresi de değişir. Bu sebepten ötürü Neelands (2004), dramanın dönüştürücü gücünü şöyle tarif eder: Drama deneyimi sayesinde kendisine ortak bir kültür oluşturan grup, küçük bir topluluk hâline gelir. Kişiler, yaratıcı dramada edindiği deneyimleri dış dünyayla olan iletişimi esnasında da kullanmaya başlar ve böylece dönüşüm meydana gelir. Sosyal alanda ve eğitim alanında bir kurum ortamının dramayla oluşturduğu sayısız deneyim vardır (bunun örneklerini Dickinson, R., Neelands, J. and Shenton İlköğretim Okulu, 2006'da bulabiliriz). Eğitimde Araştırmacı Drama bülteni (Research Drama in Education) (özellikle 13: 2 sayısı) bireysel değişimlerin çok belirgin olduğu, bireyin yaşama zevkini yeniden kazandığı, toplumu yeniden yapılandırmayı yeniden arzuladığı (buna dair bir örnek Hazou, 2008'de bulunmaktadır) sosyal alanda büyük ilgi gören eğitsel deneyimleri derlemişstir. $\mathrm{Bu}$ doğrultuda dramanın etkinliğini farklı deneyimler yoluyla toplumsal müdahale için ortaya koyan Balfour ve Somers tarafından yayımlanan kitabı (2006) tavsiye edebiliriz.

Yaratıcı dramada sürekli olarak gerçekliği sınarız ve birbirimizle çalışarak onu nasıl dönüştürebileceğimizi deneyimleriz. Gerçekliği değiştirebilecek olmanın verdiği deneyim, değişime olan inancın ortaya çıkmasındaki asli noktadır. Diğer taraftan drama oturumlarında gerçekten demokratik olan bir deneyimi yaşamak gerçek yaşamda demokrasinin nasıl olabileceğinin âdeta anlık bir görüntüsünü verir; süreç içindeki tartışmalar esnasında grup üyelerinin her birinin söyleyecek bir sözü olduğunu görmek, katılımlarına tanık olmak ve herkesin katıldığı ortak fikirleri, çalışmaları, sorumlulukları göz önünde bulundurarak yeni, müşterek bir gerçekliğin yapılandırılması bu sürecin bir parçasıdır.

Boal (1982), doğru kullanıldığı takdirde dramanın özgürleşme için etkin bir silah olduğuna kanaat getirmiştir, biz de aynı durumun eğitim için geçerli olduğuna inanıyoruz. Yaratıcı drama yoluyla gerçeklik hakkında sadece konuşmakla kalmıyoruz, böylece onu dolaylı şekilde değiştirebiliyoruz. Dramayla gerçekliği doğrudan dönüştürmüyoruz ama drama sayesinde içinde yaşayabileceğimiz gerçeklik denemeleri yapıp tecrübeler kazanabiliyoruz (Mato, 2006). Bu doğrultuda Neelands (2007), grubun kendi deneyimlerinden yola çıkarak hayali bağlamlarla çalışmasının, gerçekliğin değişim ve dönüştürmesinde etkili olduğunu, süreçte dramanın bir 
araç olarak kullanıldığını savunur. Hepimiz farkındayız ki değişim üzerine çalışmak bile bu konunun pratiğini yapanların üzerinde gerçek bir değişime sebebiyet verir. Böylece dönüşümün mümkün olduğu fikrine kalpten inanırız. Ya da Freire'in sıklıkla ifade ettiği gibi söyleyelim; "Drama değişime giden yolun umut kapısıdır."

Neelands'in belirttiği gibi (2004), bu sürecin oluşum sebebi bizim önceki tercihlerimiz ve hedeflediğimiz amaçlar, her yaşa uygun olması ve dönüşümleri kışkırtmasıdır. Bu makalenin başında da belirttiğimiz gibi eğitimde yaratıcı drama, doğası gereği tarafsız, yansız bir araç değildir. Bunun tersine, uygulanan pedagojiye olduğu kadar onu kullanan kişiye ve kişinin emellerine bağlıdır. Bunun sonucunda, Freire (1990, 2005)'in fikriyle de örtüştüğü gibi bu dönüşümün meydana gelmesi için bir basamağa daha ihtiyaç duyduğumuzu göz ardı edemeyiz: Bağlılık. Bağlılık sayesinde bir şey ya da bir kişiyi önemsediğimizi açıkça kabul ederiz, çabamızı, zamanımızı, dikkatimizi ona vakfederiz. Yaratıcı dramayı sözünü ettiğimiz bu bağlılıktan yola çıkarak kullanmak isteriz, insanlardan azından ortak bir sorumluluk bilinci ve herkes için bir dünya oluşturma arzusunu meydana getirebiliriz.

\section{Eğitimde Yaratıcı Dramanın Dönüştürücü Metodolojisi}

Şimdi de, her ne kadar farklı biçimlerde ya da farklı tasarılarda ve uygulanan seviyelere göre derinliği değişse de yaratıcı dramanın temelinde yatan bazı kilit noktaları açıklayacağız. Yaratıcı dramadan başlayarak önerdiğimiz bazı metodolojik elementler dayanışmaya ihtiyaç duyan küreselleşen dünyada özgürlüğü ve sorumluluğu desteklemede adeta doğal bir köprü vazifesini taşıyor ve bizim niyetimiz bunu göstermek.

\section{Grup: Dinlemeyi öğrenmek.}

Diğer ortamlarda bahsetmiş olduğumuz gibi (Navarro, 2006, 2008a ve 2008b) grup, eğitimde yaratıcı dramanın temellerini oluşturur. Çok kısa bir sürede fark ederiz ki, grup içerisindeki yaratıcılık her bir katılımcının katkısıyla çoğalır ve bireysel olarak hayal edilmesi bile imkânsız gibi görünen bir olgu bir de bakılır ki grup tarafından müşterek olarak başarılmış bile. Bu başarı, bireysel katkıların ötesinde müşterek bir çalışmanın benzersiz sürecinde gerçekleşir. Bu yüzden canlandırma için ilk gereksinim bu aktivitenin temeli olan, eğitsel amaçlar için kullanıldığında güçlü sosyal boyutlar elde ettiğimiz gruptur.

Bir grupla çalışmaya başladığımızda ilk yaratıcı drama oturumunda öğrenilmesi gereken kazanımların biri dinleme olmadan iletişimin mümkün olmayacağıdır; diğer bir taraftan diğer insanları ne kadar nadiren dinlediğimizin farkındalığını yaratmaktır. Bize gore dinleme; anlamayı sağlayan, kişilerin kendilerine en yakın gelen gerçekliği gözlemlemelerini, 
içine iletişim katmalarını sağlayan aktif bir tutumdur. Mato (2004)'nun açıkladığı gibi “bizim iletişimimiz ister istemez dinleme ile başlar, başkalarını dinlemek, dinyayı dinlemek, gerçekliği olduğu gibi dinlemek, hoşuma gitse de gitmese de, beni mutlu etse de mutsuz etse de dinlemekle başlar. Tüm iletişim dinleme ile meydana gelmelidir. Dinleme bizi gerçekçi yapar, rüyadan uyandırır, en ideali, bizi daha insan kılar ve bize daha çok güç verir. Her kim dinlerse farkına varır ${ }^{4}$ ve kendini bilir, kendini dönüştürür böylece dünyayı dönüştürür."

Dinleme, yaratıcı dramada takım olarak çalışabilmek için gerekli bir beceridir (Motos, 2005). Dinleme, dışarıda var olan gerçekliği drama oturumuna taşır ve bizi bu gerçekliğin varlığı konusunda farkındalık sahibi yapar böylece gerçekliğe aşina oluruz.

Ancak grup içerisinde dinlemeyi sağlayabilmek için grubun keşifler yapmasına olanak sağlayacak bir güven ortamına ihtiyaç vardır.

\section{Başarı: Güven Ortamı}

Bir grubun yaratıcı dramayı kullanırken ulaşması gereken ilk amaçlardan bir tanesi herkesin kendini rahatça ifade edebileceği ve iletişim kurabileceği tam bir güven ortamının oluşmasıdır. Özgüven ve diğer bireylere duyulan güven, 'Saçmalıyorum.' korkusunun oyunlarla birlikte aşılması bu amaca giden temel unsurdur. Güven kişiye kararlı bir umut verirken kişinin kendinden emin olmasına da yol açar. İşte bu güven ortamı ifadeyi, anlatımı, iletişimi doğurur, grup tarafından kabul görme deneyimi sırasında kişinin, gerçek karakterini göstermesine olanak sağlar.

Diğer insanlara güvenmenin temel koşullarından biri diğer kişilerin bize saygı duyduğunu, bizi önemsediğini bilmek, onlara emanet ettiğimiz şeyin bilincinde olduklarını bilmektir. Aksi hâlde, iletişim karş1lıklı değilse, güven ortadan kaybolur. Bu yüzden grup içindeki her birey diğer grup üyelerinden sorumludur, kişiler grup arkadaşları ya da kendileri hakkındaki önyargılarını ve basmakalıp fikirlerini ortadan kaldırmalıdır.

Özgürlük, sorumluluk, ve eğitimde yaratıcı drama yolunun amaçları bakımından, dinlemeyi geliştirmek, güven çalışmaları ve stratejileri çok mühimdir.

\section{Oyun}

Oyun oynadığımız zaman, tüm varlığımız işin içine dâhil olur. Sadece bilişsel düzeyde değil, hem duygularımız hem de bedenimiz işin içindedir (Moyles, 1990; Winston and Tandy, 2001; Eisnes and Mantovani, 1997). Oyun çocukların bilgiyi edinmesinde ve öğrenmesindeki temel araçtır. Sembolik oyun

\footnotetext{
4“Drama çalışması sürecine katılan herkesi bilinçli ve aktif dinlemek gerçekten özgürleștirici olan iletişim becerilerini geliştirir." (Mato, 2004)-Eğitimde yaratıcı drama dünyasıyla tanışmamız işte bu tarz bir çalışmanın tohumlarıyla başladı. Onyedi yıldan daha uzun bir zaman önce başladık ve yıllarca İspanyảdaki sokaklara, üniversitelere gittik, güneyin kuzey tarafından yağmalanması, medyanın güdümlemeleri, çocuk köleliği, göçmenlik gibi toplumsal trajedileri kınayansosyal oyunlar sahneledik... aynı zamanda gerçekte olmuş dönüşüm hikayelerini, deneyimlerini de paylaştık.
} 
ile, çocuklar çevrelerindeki gerçekliği yorumlar ve onu yeniden kurmaya ve değiştirmeye çalışırlar.

Aynı şey biz yetişkinlerin de başına gelir, gerçekliği rahat bir ortamda oyun içine oturturuz ve gerçek hayatta “acaba sonunda ne olur?” kaygısıyla asla cüret edemediğimiz yollar, davranışlar deneriz. Oyun esnasinda ortak deneyimler sayesinde, varolan durumun yansilamalarını, alt metnini okumamız vb. sayesinde gerçeği öğreniriz. Oyun, yaratıcılı̆̆ı desteklemesinin yanısıra diğer kişiler tarafından yargılanma korkusu olmaksızın rahat ve güvenilir bir ortamda çalışma ortamı sağladığ 1 için eğitimde yaratıcı dramanın çok önemli bir parçasıdır. Besbelli ki, oyun; çocuklara yönelik yaratıcı drama oturumlarında çok sık kullanılır, ve yetişkinlerle yapılan çalışmalar esnasında diğer stratejilerle harmanlanır.

\section{Yaratıcılık ve hayal gücü}

Yaratıcılık ve hayal gücü güçlükle uyarılan, belli birtakım tekniklerle geliştirilmeyi bekleyen yeteneklerimizdir.

Onlar, bizim imgeler yaratmamızı sağlar. Süreç içerisinde oluşturmaya çalıştırdığımız eğlenceli ve güven dolu ortam yaratıcılığımızı kullanmamız için bir vesile olur. Birçok kez insanlar hayal etmiş oldukları şeylere şaşırırken bu anlamlı, dışavurumcu etkinliğe devam etmek için içlerinde bir heves uyanır.

Yaratıcılık ve hayal gücü gerçeklikle daima bir iletişim içerisindedir, çünkü netiecede biz bugüne kadar toplamış olduğumuz deneyimler üzerinden yaratıcı oluruz ya da hayal kurarız (Vygoski, 1996) ve bu deneyim bize daha önce hiç görmediğimiz gerçekliği hayal etme ve anlama fırsatı sağlar. Bu yüzdendir ki, özellikle de amacımız küreselleşen dünyanın sonuçlarına yanıt aramaksa, yaratıcılık ve hayalgücü bireyler için çok önemli olan aktivitelerdir.

Oturum müşterek bir doğaçlamayla yapılır; önce kişiler birbirlerini dinler, süreç sırasındaki duygu durumu asla "hiçbir şey değiştirilemez" olmamalıdır, bunun yerine "sorunlara karşı bir çözüm bulabiliriz" inancı ve bunun kanıtlanmasıyla müşterek bir doğaçlama yapılır.

Yaratıcılık bireylerin özgür olması, gerçek hayatta karşılaşılan problemlere çözüm bulunabilmesi ya da varolan çözümlere yeni alternatifler eklenmesinin sağlanması açısından çok mühimdir. Sosyal gelişim bakış açımızdan, bu nokta belli başlı kuraldır. Yaratıcılık ve hayal gücü genellikle grup tarafından zenginleştirilir.

\section{Diğer insanları keşfetmek: saygı, işbirliği ve bă̆lılık}

Yaratıcı drama çalışmalarıyla diğer insanlara, çeşitliliğe olan saygıdeğerleri öğretimine ulaşırız (Grady, 2000). Bu da yazarların yaratıcı dramanın etik eğitiminde kullanılması potansiyalini fark etmelerini ve bu konuda derin çalışmalar yapmalarını sağlamıştır (Winston, 2000). Dramatizasyon insanların sahip oldukları şeyler için değil, salt insan oldukları için değerlerini, kim- 
liklerini kavramak için eğitimde firsat sağlar.

Mallika (2000) yaratıcı dramayı öğrenme metodlarında bu öğrenmenin yönlendirici olduğunu açıklar. Böylece yaratıcı dramayla kendimizi bir karakterin rolü içine koyarız, belirli bir bağlamda belirli bir durumu ele alırız, karakterin duygularını, olası ahlaki ikilemlerini ele alırız; onların hayatlarındaki doğruları tüm bağlılığımızla bulmaya çalışırken bizimkinden farklı olan diğer yaşamları anlamaya ve onlara değer vermeye çalışırız. Yaratıcı drama diğer insanların doğrularını görmemizde bize yeni bakış açıları sağlar. Çünkü bir rolü üzerimize giydiğimizde “Kendimizden yola çıkarak diğer kişi oluyoruz.” (Neelands, 2002). Bu da bizim görüşlerimizden farklı olan bakış açılarını keşfederek, kendi bakış açımızı zenginleştirmemizi sağlar.

\section{Deneyimin ve aktarımın değerlendirilmesi}

Her drama oturumunda değerlendirme bölümü gerekli bir unsurdur. Bu ise deneyimlerimizin birbirine katışmasıyla, bizim herbirimizin gerçeğiyle ve dünyanın gerçeğiyle ne denli ilişkili olduğunu yansıtmamızla ortaya çıkar. Bu an, oturumdaki her katılımcının gerçeği kendi perspektifinden yansıttığ1 ve bizim derinlemesine düşünmemizi sağladığg için çok önemli bir andır. Süreç boyunca birçok deneyim paylaşılmış, belirli deneyimler gerçekliğe transfer edilmiştir.

Neeland'1, "Yaratıcı drama, bir demokrasi pratiğidir ya da değişim için yapılan bir pratiktir." demeye iten şey, yaratıcı dramanın gücüdür (2007). Kurgusal deneyimimiz sayesinde, değerlendirme sonunda, gerçek hayatta yaşamış olduğumuz deneyimin farkındalığını kazanırız ve bu sırada gerçekliğin bir parçasının herkes tarafından inşa edildiğini ve bizim gerçeğimizle bağlantılı olduğunu da görürüz. Biz gerçekliğin dönüşümüne dair temeller kurmaya çalışırken değerlendirme sırasında geçerli olan bazı deneyimleri tercih etmeli, onları dünyanın durumuyla ve kendi gerçekliğimizle bağdaştırmalıyız.

Eylem-yansıma-eylem, yaratıcı drama oturumlarında bu süreci takip ederiz. Öğrenme deneyimseldir (Motos, 2000), biz olaya ne kadar katılırsak, fikirlerimizi, duygularımızı, ruhumuzu, bedenimizi, deneyimlerimizi belirli bir bağlama ne kadar dahil edersek, eyleme geçme konusunda o kadar çok deneyim kazanırız.

\section{Sonuç}

Eğitimin hepimiz için fayda sağlayan bir sorumluluk bilinciyle yürütülmesi gerekliliği acilen anlaşılması gereken önemli bir konudur.

Eğitimde yaratıcı drama yaşam için önemli öğretiler sunar, örneğin, grubun uygunluğu, diğer insanları keşfetmek ve onlarla bağlantı kurmak gibi. Drama yoluyla güven dolu bir ortamda, aynı amaç uğruna çalıştığımız takdirde diğer insanların bizi zenginleştirdiğini ve bizim kapasitemizi dönüştürebildiğini deneyimleriz. Bu tip fikirleri teşvik etme bakış açımızla, bizim 
için öğrenme asli olan şeydir. Diğer insanlarla ilişki kurabilmek, onları desteklemek; sosyal değişiklikler için, değişim karşısındaki güçsüzlügüumüzü yenmek ve hayatımıza azimle devam etmek için inanılmaz mühimdir.

Ancak bu yolda, eğitimde yaratıcı drama sadece bir araçtır, bu yüzden onun kısıtlamalarının farkındayız. Salt yaratıcı dramayla özgür bireyler yetiştiremeyiz. Ancak, her ne kadar nadiren keşfedilse ve değerlendirilse de, yaratıcı drama dayanışma perspektifiyle kullanıldığında, çok güçlü bir potansiyale sahiptir. Değişim ve dayanışmanın gelişimi için bizim tutumumuz barışın ve demokrasinin gerçek anlamda gelişmesidir, öyle bireyler, vatandaşlar yetiştirmek istiyoruz ki, onlar geleceğimizin umut $1 s ̧ ı$ ğı olsun.

\section{Kaynaklar}

Alumnos de la escuela de Barbiana (1970). Carta a una maestra. Barcelona: Nova Terra.

Balfour, M. and Somers, J. (Eds) (2006). Drama as social intervention. North York: Captus University.

Bernabeu Rico, J. L. (2006). Las teorías personalistas. In A. J. Colom; J.L. B; and J. Sarramona (Eds.), Teoría de las Instituciones Educativas Contemporáneas (6th ed.)(pp. 125-135). Barcelona:Ariel.

Boal, A. (1982). Teatro del oprimido. México: Nueva Imagen.

Dickinson, R., Neelands, J. and Shenton Primary School (2006). Improve your Primary School through drama. Gran Bretaña: David Fulton Publishers.

Eines, J. and Mantovani, A. (1997). Didáctica de la Dramatización. Barcelona: Gedisa.

Freire, P. (1990). La naturaleza política de la educación. Barcelona: Paidós.

Freire, P. (2005). Pedagogía de la esperanza. Un reencuentro con la pedagogía del oprimido. (6th Ed.) México: Siglo XXI.

González Monteagudo, J. (2003). Ser Educador en un Mundo globalizado. La Educación en el Siglo XXI. Ciclo de Conferencias. Seville: Ayuntamiento de Sevilla, 23-36.

Grady, S. (2000). Drama and Diversity. A pluralistic perspective for educational drama. Portsmourth: Heinemann.

Guiso Medellín, A. (2003). Cinco claves éticos-políticas de Freire.www.solidaridad.net, Education Section. Date: 2008-08-15.

Hazou, R. (2008). Refugitive and the theatre of dys-appearance. Research in Drama Education, 13 (2), 181-186.

Mallika, H. (2000). Drama's Ways of Learning. Research in Drama Education, 5:1, 45-62.

Mato López, M. (2004). Teatro de la escucha: Una herramienta para la promoción. www.teatroycompromiso.com. Date: 2008-08-4. 
Mato, M. (2006). El baúl mágico. Imaginación y creatividad con niños de 4 a 7 años. Ciudad Real: Ñaque.

Motos, T. (2000): Aprendizaje vivencial. In F. Bercebal; D. de Prado; G. Laferrière and T. Motos (Eds.), Con los pedagogos de hoy. Sesiones de trabajo (pp.134-156).Ciudad Real: Ñaque.

Motos Teruel, T. (2005). Expresión total y educación emocional. Recrearte.http://www.iacat. Com/1Cientifica/tomas.html Section: Creativity in Education. Date: 2008-07-2.

Moyles, J.R. (1990). El juego en la educación infantil y primaria. Madrid: Morata.

Navarro Solano, M.R. (2005). El valor pedagógico de la dramatización: su importancia en la formación inicial del profesorado. PhD Thesis.Seville University.

Navarro Solano, M.R. (2006). Prólogo. In M. Mato, El baúl mágico. Imaginación y creatividad con niños de 4 a 7 años (pp. 9-14). Ciudad Real: Ñaque.

Navarro Solano, M.R. (2008a). Drama, educación y compromiso, "In press".

Navarro Solano, M.R. (2008b). La educación de la afectividad humana a través de la dramatización. In C. Romero Pérez and L. Núñez Cubero, (Ed.): Seville: Servicio de Publicaciones de la Universidad de Sevilla. "In press"

Neelands, J. (2004). Miracles are happening: beyond the rhetoric of transformation in the Western traditions of drama education. Research in Drama Education, 9 (1), 47-56.

Neelands, J. (2002). 11/09-The Space in our Hearts. Drama (Summer), 4-10.

Neelands, J. (2007). Acting together; ensemble as democratic process in art and life, "in press".

Núñez Cubero, L. and Romero, C. (2003). Pensar la Educación: conceptos y opciones fundamentales. Madrid: Pirámide.

United Nations Children's Fund (UNICEF) (2008). Estado Mundial de la Infancia 2008. Nueva York: Sección de Edición y Publicaciones de UNICEF.

Vygotski, L. S. (1996). La imaginación y el arte en la infancia. (3rd Ed.). Madrid: Akal.

Winston, J. (1999). Theorising Drama as Moral Education. Journal of Moral Education, 28 (4), 459-471.

Winston, J. (2000). Drama, Literacy and Moral Education 5-11. London: David Fulton.

Winston, J. and Tandy, M. (2001): Beginning Drama 4-11. (2nd Ed.)London: David Fulton Publishers.

Ziegler, J. (2007). El Imperio de la Vergüenza. El orden mundial 'asesina' cada día a 100.000 personas hambrientas (extracto).www.solidaridad.net.Section Hunger. Date: 2007-10-19. 



\title{
Drama in Education and Social Change: Theoretical Aspects
}

\author{
$M^{\text {a }}$. Rosario NAVARRO \\ University of Seville
}

\begin{abstract}
Drama in education is a tool that allows the explanation of aspects of reality that are difficult to address with other educational methods. In particular, it becomes a powerful tool for personal and social development for all those involved. Here we discuss the possibilities of drama for promoting personal and collective social responsibility in children, young people and adults, for example, training in citizenship for a just world that is truly democratic. However, it is common for drama to be used with scant attention to any underlying educational techniques. Here we discuss some key issues that show some theoretical aspects of drama in relation to social development and social change and how its associated techniques -which are very well-defined and thus require careful training-are closely related to fundamental subjects of the aims and values that underpin the whole practice of education.
\end{abstract}

Keywords: Drama in education, social responsibility, creativity, group and social change.

\section{$\ddot{O}_{z e t}$}

Ĕ̆itimde drama, gerçek durumları tanımlamayı sağlayan bir araçtır, bu durumların saptanması diğer ĕgitim yöntemleriyle zordur. Özellikle de, drama sürecine giren herkesin kişisel ve sosyal gelişimi için çok güçlü bir araç hâline gelir.Bu makalede çocuklarda, gençlerde, yetişkinlerde kişisel ve müş̧terek sosyal sorumluluğu teşvik etmede yaratıcı dramanın nasıl etkili olabileceğini tartıştık. Örneğin, tamamen demokratik olan adil bir dünya için vatandaşlık eğitimi kavramı bunlardan birisidir. Ancak, yaratıcı dramanın temel ĕgitim teknikleriyle dar bir alanda kullanılması yaygındır. Bu makalede dramayla sosyal gelişim, sosyal değişimin teorik açılardan ilişkilerinin kilit noktalarını tartışıoruz. Ayrıca dramayla bağlantılı-son derece iyi tanımlanmış ve böylece dikkatli bir eğitim gerektiren- teknikler bütün eğitim pratiğini tümüyle destekleyen amaçların esaslı konuları ve değerleriyle yakından ilişkilidir.

Anahtar Sözcükler: Eğitimde drama, sosyal sorumluluk, yaratıcılık, grup değişimi ve sosyal değişim.

\footnotetext{
${ }^{1}$ Associated Professor of Seville University (Spain). Dr. in Science Education. Degree in Science Education and Philosophy at Sevilla University Her PhD thesis (awarded as European Doctorate and best PhD Thesis 2005 at Seville University) was titled The pedagogical value of Drama in Education: its importance in the initial teacher training. rosanavarro@us.es
} 


\section{Thinking About Education: The Matter of Aims}

As educators we need to define the framework of values from which we act, our view of the human being, the vision of reality we seek to respond to and the aims we pursue, which will greatly depend on our analysis of reality, as well as our standpoint to understand it. Everyone chooses a standpoint with regard to reality which conditions the way we act, consequently we must be aware of ours and its consequences for our students and for society.

Therefore, every educational action needs and is based upon philosophical foundations, closely related to the set of values that orientate our teaching task. These philosophical foundations constitute the framework that leads us to choose certain methods and means to achieve the aims we have set ourselves, and conversely, the aims we have established have a direct impact on how we plan our educational activity. Thus, there is no such thing as a neutral education activity, for there is always a purpose and an aim (Núñez and Romero, 2003).

As any other educational practice, the use of drama in education is not neutral either, as it depends on the intentions of the person utilising it, on their idea of mankind, as well as on the culture within which it takes place (Neelands, 2004; Motos, 2006). It is, therefore, of the utmost importance to try to objectivise the reality to which we are responding and to support our aims with arguments.

In this paper, and partly using the English currents on Drama in Education, we seek to point out some aspects we consider fundamental of the use of drama in the full training of individuals, which is ultimately the essential goal to which the educational task wishes to contribute.

\section{Educational Aims and Personalistic Trends in Education}

We consider individuals as free but not isolated beings, as essentially social, transcendental in their own worth, which prevents them from becoming mere objects. We are aware of the fact that the incorruptible value of the human dignity of each individual is being questioned, with facts, in Western culture.

Furthermore, we understand that freedom goes hand in hand with responsibility; freedom as conceived by Mounier (Bernabeu, 2006), which on the one hand requires personal responsibility and on the other unavoidable commitment to others. Thus, the freer we are the more able we are choose those alternatives that are most in tune with our human nature, one of which is our social being, which leads us to need others and to become committed to others.

I short, freedom, dignity, our social nature, our responsibility to others or our commitment are fundamental premises on which the essence of our identity is based, and to ignore them or avoid them goes against our own nature. Starting from our identification with personalistic trends in education (Mounier 1905-1950, Milani 1923-1967, Freire 1921-1997), we understand that the essential realisation of an individual is their freedom, which requires an autonomous conscience that will allow them to establish a relationship with the world through knowledge and action (Bernabeu, 2006). Therefore, we maintain in our educational proposal the characteristics 
of this personalistic view of education which are given by its communitarian nature, which is open to society, active and critical, fostering democratic participation and, ultimately, pursuing the full and collective development of human beings as the aim of education.

In this research paper we try to briefly describe some essential aspects of drama practice in education which contribute to the aims we have established.

\section{Contextualising Education}

Our analysis of reality partly determines our work in education. For this reason, we believe it is most important to reflect on this point.

Milani in his teachings used to start from the most concrete reality that worried his students to open their horizons to the rest of the world. Education emerged from life, but did not remain within the personal or group area, not even in the local or national milieu, but incorporated the classroom to the world situation, which demanded taking a stand. The importance that Milani gave to the collective reading of newspapers or to history lessons is well known. These specific activities were the starting points for the search for the causes of conflicts, both from a historical and a political viewpoint (Students from the Barbiana School, 1970). Education had clear a social and political dimension. For Milani, as for Freire (1990), it is through education that we train the citizens of the future, who have the power to confront reality and transform it, for which they need to seriously reflect on it.

Like them, we too believe that every educational process is contextualised within a specific historic moment and needs to be inserted in it. Through education we try to train people to be in control and to respond to the specific society in which they have to live, in order to construct a fairer and more compassionate world.

Our historic moment is conditioned by the globalisation process, which has become one of the key concepts in the general description of the world at present. One of its best known characteristics is the interconnection of a large part of the world. However, the most decisive fact of globalisation is the existence of vast starving crowds, who in various degrees of poverty, make up the majority of humankind ${ }^{2}$. We agree with González Monteagudo (2003) when he states, analysing the characteristics of globalisation that "what has been globalised is not development, but inequality". Starvation is the most common way to die in our world nowadays. This outrageous, terrible and inhumane reality takes place day after day in front of our eyes and our conscience, leaving us helpless to look for answers ${ }^{3}$. This is actually the everyday reality in which the life of the majority of the population of this planet takes place. We are confronted with a reality that has acquired the status of a real social issue throughout the world.

\footnotetext{
${ }^{2}$ The United Nations Organitation for Children (UNICEF, 2008) says that "the problem of hunger is associated until 50\% of infant death in the world".

${ }^{3}$ Jean Ziegler, old Vicepresident of the World Bank and nowadays Counselor of the United Nations for food subjects, affirms that "we are opposite a deliberate massacre, which happens day after day, under our iced nonchalance. Hunger has not been a thing of destination from a long time ago. It supposes a silent mass murder". (Ziegler, 2007).
} 
In contrast with this world situation, our lives go on as if human suffering did not exist, as if it did not concern us. We consider conscience manipulation on this issue to be a fraud against each individual to whom this reality is denied. Every man and woman has the right to be able to decide, in the exercise of their freedom, which attitude to take to a globalised world and to an oppressed majority. For this reason, we believe there is a need to generate spaces where values and attitudes of respect for human beings and a desire to defend their rights are taught and learnt. Likewise, we maintain that it is crucial to develop creativity to allow us to devise new responses to the unknown. From this standpoint, we understand that drama in education is an appropriate educational tool to foster these spaces for the learning of morals and values (Winston, 1999).

\section{The Need for Another Reality}

Our aim is not just to understand reality, we also seek to change it. In the face of reality as we have described it, our freedom, experienced from our responsibility, drives us to take charge of the situation. We identify with the term used in Freire's pedagogy "hopeful realism" (Freire, 2005). This situation, far from being pessimistic, leads to an active attitude to the construction of a fairer reality, that is to say, it is this analysis of reality which convinces us of the urgent need to change it, and to the certain hope and firm belief in this change. As explained in Guiso Medellín (2003) Freire understands hope as a necessary but insufficient element. Hope by itself does not transform the world, but it is not possible to transform it without it, if we want to do it. Without hope we cannot even start changing processes, but without these processes, hope is corrupted and becomes "tragic desperation", and hopelessness becomes the same as resistance to change and immobility. Hope needs facts to become historical reality (Freire, 1992 in Guiso Medellín, 2003). We often observe in the generations of students we teach a profound lack of hope with regard to the possibility of changing reality, which they watch impassively and which, naturally, affects them to a very great extent.

Through education we look for ways to make this transformation of reality possible. Evidently, a clear choice is required on the part of educators in favour of this change both in the classroom and outside.

\section{Drama and Transformation}

Our nature as social beings possesses a fundamental characteristic that leads us to take responsibility of close and distant reality.

The transforming force of drama in education stems on the one hand from the changes it brings about in the individual him/herself, unblocking expression, enhancing self-confidence, encouraging trust in others, developing a capacity for listening and discussing; and on the other hand, a change in the individual causes their environment to change as well. Therefore, Neelands (2004) states that the transforming force of drama lies in the fact that from the drama experience a group builds a common culture and generates a small community, which later spreads to the rest of their relationships outside the drama sessions. There are numerous experiences in the social and educational field, in which the environment of an institution is transformed through 
drama (we find an example of this in Dickinson, R., Neelands, J. and Shenton Primary School, 2006). The journal Research Drama in Education (especially number 13: 2) has gathered recently different educational experiences of great interest in the social field, where change in individuals is obvious, making them regain their zest for life and their desire to construct society (an example can be found in Hazou, 2008). Along these lines, we also recommend the book published by Balfour and Somers (2006) which presents the effectiveness of drama for social intervention through different experiences.

In drama we are permanently testing reality as it comes, and we experience how working together we can transform it. This experience of being able to change reality is essential to create a belief in change. On the other hand, living a truly democratic experience in drama sessions gives us a glimpse of what democracy in real life would be, for in discussions and listening each member of the group has something to say and to contribute, and through everyone's shared ideas, work and commitment a new reality can be constructed jointly.

Following the conviction that led Boal (1982) to think that drama is an effective weapon for liberation if used appropriately, we believe that this also happens in education. For with drama, not only do we speak about reality, we can also change it indirectly. Through drama we do not transform reality directly, but we make it possible to carry out trials and tests that will enable us to live in it (Mato, 2006). Along these lines, Neelands (2007) maintains that drama is an indirect instrument to bring about change and transformation in reality by working with imaginary contexts devised by the group after their experience incarnated in reality. We are aware of the fact that working towards change already brings about a real transformation in those who practise it. We thus become immersed in the logic that transformation is possible. That is to say, it opens a door to hope, as Freire often declares.

As Neelands (2004) states, this occurs because our previous option and aim seek, adapted to each age, to provoke these transformations. Drama in education is not a "neutral" tool as we pointed out at the beginning of this article, with characteristics associated to its nature, as it is sometimes presented. On the contrary, it depends on the person using it and their aims, as well as on the pedagogy employed. Thus, and coinciding with Freire (1990, 2005), we cannot ignore that for this transformation to take place we need one more step: commitment. Through commitment we firmly decide that we care about something or somebody, we devote our effort, time or attention to them. We want to use drama from this commitment, in the knowledge that we can at least aspire to generate in others the desire to look for ways to materialise this communal responsibility of constructing a world for all

\section{The Transforming Methodology of Drama in Education}

We shall now explain some of the keys that lie at the bottom of drama work, although in different forms and with different proposals and varying in depth according to the stages at which it is implemented. It is our intention to show that some of the methodological elements we propose starting from drama can be linked naturally with promoting freedom and responsibility 
in a globalised world that is in need of solidarity.

\section{The Group: Learning To Listen}

As we have mentioned on other occasions (Navarro, 2006, 2008a and 2008b) the group constitutes the basis for drama work in education. Within a short period of time we understand that creativity is multiplied with everyone's contributions and what would be impossible to imagine on an individual level is progressively achieved communally, beyond individual contributions, in a unique process of collective construction. Therefore, the first requirement for dramatisation is a group as the basis for activity, from which we derive their strong social dimension when utilised for educational objectives.

One of the objectives in the first drama sessions when we start work with a group is to make them understand that communication is impossible without listening; and on the other hand, to make them realise how little we usually listen to others. We understand listening as an active attitude that enables individuals to understand, observe the reality that is closest to them, adding communication to it. As Mato explains (2004)

"our communication starts necessarily from listening, listening to others, listening to the world, to reality as it is, whether I like it or not, whether it makes me sad or happy. All communication should arise from listening. Listening makes us realistic and awakens in us the dream, the ideal, making us more human and giving us greater power. He who listens becomes aware 4 and knows himself, transforms himself and is able to bring about change". 3

Listening is a necessary skill for working as a team in drama (Motos, 2005). Listening brings the reality outside into drama sessions and makes us aware of its existence, which is essential to become familiar with it.

However, to make listening possible within a group we need an atmosphere of trust that will allow us to explore.

\section{Achievement: An Atmosphere of Trust}

One of the first aspects that a group needs to attain when using drama is to generate an atmosphere of absolute trust to be able to express oneself and to communicate. Self-confidence and trust in the other members of the group, overcoming fear of ridicule, with play as a key element to attain this goal. Trust entails both putting firm hope in a person and being sure of oneself. It is this atmosphere of trust that makes expression, communication and showing one's true personality possible, whilst undergoing the experience of being accepted by the group.

One of the bases for trusting others is feeling their respect for us, attention to us and their

\footnotetext{
4"Listening drama works towards the conscious and active protagonism of all those who participate in this process and moves ahead with the objective of developing communication strategies that are really liberating" (Mato, 2004)- Our introduction to the world of drama in education started with the seeds of this type of work, over seventeen years ago and for years we went through streets and universities throughout Spain, presenting social plays in which we denounced publicly tragedies such as the plundering of the south by the north, the manipulation of the media, child slavery, immigration..., as well as transformation experiences which actually happened in reality.
} 
responsibility for what we entrust them with. Otherwise, if communication is not reciprocated, trust disappears. It is, therefore, of the utmost importance that everyone should feel responsible for everyone else and should be able to do away with the prejudices and stereotypes that we have about others and about ourselves.

With regard to the goals of freedom, responsibility and commitment through the use of drama in education, practising listening and confidence techniques and strategies is essential.

\section{Play}

When we play, our whole being becomes involved, not only our cognition and emotion but also our body (Moyles, 1990; Winston and Tandy, 2001; Eisnes and Mantovani, 1997). Play is the main means for children to learn and acquire knowledge. Through symbolic play, children interpret the reality around them and try to reconstruct it and modify it.

The same occurs with adults when we place them in a play situation: we place them in a relaxed situation and we dare to try out behaviours and conducts that we would not be capable of practising in reality for fear of the consequences. Through play we come to know reality as we go through communal experiences, readings of situations, reflections, etc. Play is an essential part of drama in education, for, besides encouraging creativity, it fosters a favourable atmosphere to work in without fear of being judged. Evidently, play is most often present when structuring drama sessions aimed at children, and is combined with other strategies when working with adults.

\section{Creativity and Imagination}

Creativity and imagination are scarcely stimulated capacities that deserve to be developed with the aid of certain techniques. These allow us to create images. The recreational and trusting environment we seek to create throughout this process is conducive to using our imagination. On many occasions people are surprised by what they have imagined, reinforcing their will to continue exercising an expressive activity.

Creativity and imagination are in constant dialogue with reality, for we create and imagine on the bases of the experience we have accumulated (Vygotski, 1996) and it is this experience which allows us, for example, to imagine or understand realities we have never seen. Therefore, it is a highly important activity for individuals, especially if our aim is to provide new answers to the consequences of globalisation.

Training is done through communal improvisation starting from listening, from a willingness to believe and to prove that together we can find solutions, as opposed to the feeling of impotence of "nothing can be done".

Creativity is essential for individuals to be free and to find solutions to real life problems or to create new alternatives that can be added to those already existing. From our social development perspective, this point is fundamental. Creativity and imagination are generally enriched by the group. 


\section{Discovering Others: Respect, Collaboration and Commitment To Others}

From drama activities we approach the teaching of values related to respect for others and for diversity (Grady, 2000). This has led authors to notice and do an in-depth study of the potential of drama for ethical and moral education (Winston, 2000). Dramatisation presents itself as an opportunity in education to learn the worth of others, for what they are and not for what they own.

Mallika (2000) explains this learning process in her model of the ways of learning drama. Thus, through drama practice we place ourselves in the role of a character, taking on their particular situation within a specific context, their feelings and their potential moral dilemmas; we are trying to understand and value other lives different from ours from the firm commitment of searching for the truth in their lives. Drama opens up new perspectives to see the reality of others, for when we accept a role, we are "being others from our own selves" (Neelands, 2002), enriching our perspective as we explore different viewpoints which are different to ours.

\section{Experience and Transposition Assessment}

In every drama session assessment is a necessary element. This occurs when we join our experiences and together try to reflect on how this is related to our reality, the reality of each and everyone of us and that of the world. This is a crucial moment as it allows us to reflect on reality from the full receptiveness of each participant in the session -due to the experience shared in it-. It is a matter of transferring specific experience to reality.

It is the force of drama that leads authors like Neelands to state that drama is a democracy practice (2007) or a practice for change. This is because through evaluation we become aware of what we have experienced in life through an imagined experience, not forgetting that a part of reality is constructed by all and can be linked with it. As we seek to establish the foundations for a transformation of reality to occur, we shall favour certain experiences that are valid at the time of assessment, linking them with the situation of the world and of our own reality.

Action-reflection-action, this is the process we followed in education drama sessions. The learning is experiential (Motos, 2000), in as much as we all act, involving our thoughts, emotions, spirit, body and experience in a specific context, which we shall later transpose through reflection, and from this point we shall be again encouraged to act.

\section{Conclusion}

It is a matter of urgency that education should be conducted in the responsibility that all and everyone of us have towards the common good.

Drama in education offers crucial teachings for life, such as the relevance of the group, discovering others and commitment to others. Through drama we experience that others enrich us and generally transform our capacities when we work in a trusting environment towards the same end. For us, from our perspective of promoting these ideals, this learning is essential, for we are aware of the fact that associating with others and supporting them are crucial aspects for social change, for overcom- 
ing impotence when faced with change and for persevering in our chosen path.

However, drama in education is, in short, a mere tool, therefore, we are aware of its limitations. It is not a means that can by itself create free individuals. Non-the-less, when used for the promotion of a solidarity perspective we believe it has high potential, though scarcely explored or assessed. Our position in terms of the promotion of change and solidarity is an option that attempts to serve the true development of peace and democracy, to educate citizens so that they become a real hope for the future.

\section{References}

Alumnos de la escuela de Barbiana (1970). Carta a una maestra. Barcelona: Nova Terra.

Balfour, M. and Somers, J. (Eds) (2006). Drama as social intervention. North York: Captus University.

Bernabeu Rico, J. L. (2006). Las teorías personalistas. In A. J. Colom; J.L.Bernabeu; E. Domínguez and J. Sarramona (Eds.), Teoría de las Instituciones Educativas Contemporáneas (6th ed.) (pp. 125135). Barcelona: Ariel.

Boal, A. (1982). Teatro del oprimido. México: Nueva Imagen.

Dickinson, R., Neelands, J. and Shenton Primary School (2006). Improve your Primary School through drama. Gran Bretaña: David Fulton Publishers.

Eines, J. and Mantovani, A. (1997). Didáctica de la Dramatización. Barcelona: Gedisa.

Freire, P. (1990). La naturaleza política de la educación. Barcelona: Paidós.

Freire, P. (2005). Pedagogía de la esperanza. Un reencuentro con la pedagogía del oprimido. (6th Ed.) México: Siglo XXI.

González Monteagudo, J. (2003). Ser Educador en un Mundo globalizado. La Educación en el Siglo XXI. Ciclo de Conferencias. Seville: Ayuntamiento de Sevilla, 23-36.

Grady, S. (2000). Drama and Diversity. A pluralistic perspective for educational drama. Portsmourth: Heinemann.

Guiso Medellín, A. (2003). Cinco claves éticos-políticas de Freire. www.solidaridad.net, Education Section. Date: 2008- 08-15.

Hazou, R. (2008). Refugitive and the theatre of dys-appearance. Research in Drama Education, 13 (2), 181-186.

Mallika, H. (2000). Drama's Ways of Learning. Research in Drama Education, 5:1, 45-62.

Mato López, M. (2004). Teatro de la escucha: Una herramienta para la promoción. www.teatroycompromiso.com. Date: 2008-08-4.

Mato, M. (2006). El baúl mágico. Imaginación y creatividad con niños de 4 a 7 años. Ciudad Real: Ñaque. 
Motos, T. (2000): Aprendizaje vivencial. In F. Bercebal; D. de Prado; G. Laferrière and T. Motos (Eds.), Con los pedagogos de hoy. Sesiones de trabajo (pp.134-156). Ciudad Real: Ñaque.

Motos Teruel, T. (2005). Expresión total y educación emocional. Recrearte. http://www.iacat.com/1Cientifica/tomas.html . Section: Creativity in Education. Date: 2008-07-2.

Moyles, J.R. (1990). El juego en la educación infantil y primaria. Madrid: Morata.

Navarro Solano, M.R. (2005). El valor pedagógico de la dramatización: su importancia en la formación inicial del profesorado. $\mathrm{PhD}$ Thesis. Seville University.

Navarro Solano, M.R. (2006). Prólogo. In M. Mato, El baúl mágico. Imaginación y creatividad con niños de 4 a 7 años (pp. 9-14). Ciudad Real: Ñaque.

Navarro Solano, M.R. (2008a). Drama, educación y compromiso, "In press".

Navarro Solano, M.R. (2008b). La educación de la afectividad humana a través de la dramatización. In C. Romero Pérez and L. Núñez Cubero, (Ed.): Seville: Servicio de Publicaciones de la Universidad de Sevilla. "In press"

Neelands, J. (2004). Miracles are happening: beyond the rhetoric of transformation in the Western traditions of drama education. Research in Drama Education, 9 (1), 47-56.

Neelands, J. (2002). 11/09- The Space in our Hearts. Drama (Summer), 4-10.

Neelands, J. (2007). Acting together; ensemble as democratic process in art and life, "in press".

Núñez Cubero, L. and Romero, C. (2003). Pensar la Educación: conceptos y opciones fundamentales. Madrid: Pirámide.

United Nations Children's Fund (UNICEF) (2008). Estado Mundial de la Infancia 2008. Nueva York: Sección de Edición y Publicaciones de UNICEF.

Vygotski, L. S. (1996). La imaginación y el arte en la infancia. (3rd Ed.). Madrid: Akal.

Winston, J. (1999). Theorising Drama as Moral Education. Journal of Moral Education, 28 (4), 459-471.

Winston, J. (2000). Drama, Literacy and Moral Education 5-11. London: David Fulton.

Winston, J. and Tandy, M. (2001): Beginning Drama 4-11. (2nd Ed.) London: David Fulton Publishers.

Ziegler, J. (2007). El Imperio de la Vergüenza. El orden mundial 'asesina' cada día a 100.000 personas hambrientas (extracto). www.solidaridad.net. Section Hunger. Date: 2007-10-19. 